\title{
Strong Duality \\ for Transportation Flow Problems
}

\author{
R. Klötzler
}

Abstract. This paper is a supplement and correction to the author's article "Optimal transportation flows" [2]. By new methods the existence of optimal transportation flows and the strong duality to deposit problems is proved.

Keywords: Transportation flow problems, deposit problems, dual optimization problems AMS subject classification: 49 N 15, 49 Q 15, 49 Q 20

\section{Introduction}

In conformity with [2] we consider the following transportation flow problem:

$$
K(\mu):=\int_{\Omega} r(x, d \mu(x)) \rightarrow \min \quad \text { on } Y
$$

where

$$
Y:=\left\{\mu \in L_{\infty}^{n n}(\Omega)^{*} \mid\langle\nabla \sigma, \mu\rangle=K_{D}(\sigma) \quad \forall \sigma \in W_{\infty}^{1, n}(\Omega)\right\}
$$

and

$$
K_{D}(\sigma):=\int_{\Omega} \sigma(x)^{\top} d \alpha(x) \quad \text { on } W_{\infty}^{1, n}(\Omega)
$$

We assume, $\Omega$ is a bounded strongly Lipschitz domain of $E^{m}, \alpha=\left(\alpha_{1}, \ldots, \alpha_{n}\right)$ is a given vector of finite Borel measures $\alpha_{k}$ on the $\sigma$-algebra $\mathfrak{B}$ of all Lebesgue-measurable subsets of $\mathfrak{B}$ which satisfy the assumption

$$
\int_{\mathbf{\Omega}} d \alpha_{k}=0 \quad(k=1, \ldots, n)
$$

$r$ is a given local cost rate on $\Omega \times E^{m n}$ with the following basic properties:

$$
\left.\begin{array}{l}
r(\cdot, v) \text { is summable on } \Omega \\
r(x, \cdot) \text { is positive homogeneous of degree one and convex on } E^{m n} \forall x \in \Omega \\
\gamma_{1}|v| \leq r(x, v) \leq \gamma_{2}|v|\left(v \in E^{m n}, x \in \Omega\right) \text { for some constants } \gamma_{1}, \gamma_{2}>0 .
\end{array}\right\}
$$

R. Klötzler: Tauchaer Str. 202, D - 04349 Leipzig

ISSN 0232-2064 / $\$ 2.50$ (C) Heldermann Verlag Berlin 
The objective functional of (1) is defined by

$$
\int_{\Omega} r(x, d \mu(x)):=\sup _{u}\left\{(u, \mu\rangle \mid u \in L_{\infty}^{m n}(\Omega), u^{\top}(x) v \leq r(x, v) \forall v \in E^{m n}\right\} .
$$

Every element $\mu=\left(\mu_{1}, \ldots, \mu_{n}\right) \in Y$ is said to be a feasible flow and $\mu_{k}$ the flow of the $k$-th tranportation good.

Referring to [2], between the transportation flow problem (1) and the deposit problem

$$
K_{D}(S)=\int_{\Omega} S(x)^{\top} d \alpha(x) \longrightarrow \max \quad \text { on } \mathfrak{S}^{\prime}
$$

there exists duality, i.e.

$$
K(\mu) \geq K_{D}(S) \quad \forall \mu \in Y, S \in \mathfrak{S}^{\prime}
$$

if we define $\mathfrak{S}^{\prime}$ by

$$
\mathfrak{S}^{\prime}:=\left\{S \in W_{\infty}^{1, n}(\Omega) \mid \nabla S(x) \in \mathfrak{F}(x) \text { for a.e. } x \in \Omega\right\}
$$

with

$$
\mathfrak{F}(x):=\left\{z \in E^{m n} \mid z^{\top} v \leq r(x, v) \forall v \in E^{m n}\right\}
$$

The restrictions of (9) characterize slope restrictions in the sense that $\nabla S(x)$ belongs to the convex figuratrix set $\mathfrak{F}(x)$ for a.e. $x \in \Omega$.

Since (4), the linear functional $K_{D}$ has the property $K_{D}(S)=K_{D}(S+C)$ for any constant vektor $C \in E^{n}$. Therefore, without loss of generality we can reduce the deposit problem (7) on the restricted class $\mathfrak{S}:=\left\{S \in \mathfrak{S}^{\prime} \mid S(\hat{x})=0\right\}$ where $\hat{x}$ is an arbitrary fixed point in $\bar{\Omega}$.

We know from [2] the following theorem.

Theorem 1. The deposit problem (7) has an optimal solution $S_{0}$.

\section{The existence of optimal flows}

In $L_{\infty}^{m n}(\Omega)^{*}$ the standardized norm is defined by

$$
\|\mu\|:=\sup _{u}\left\{\langle u, \mu\rangle\left|u \in L_{\infty}^{m n}(\Omega),\right| u(x) \mid \leq 1 \text { a.e. on } \Omega\right\} .
$$

We introduce in this Banach space an equivalent norm by

$$
\|\mu\|^{*}:=\sup _{u}\left\{\langle u, \mu\rangle \mid u \in L_{\infty}^{m n}(\Omega), u(x) \in \mathfrak{F}(x) \text { a.e. on } \Omega\right\} .
$$


The equivalence of both norms is obvious under consideration of the third property of assumption (5):

$$
\begin{aligned}
\sup _{u} & \left.\{u, \mu\rangle\left|u \in L_{\infty}^{m n}(\Omega), u(x)^{\top} v \leq \gamma_{1}\right| v \mid \forall v \in E^{m n}, \text { a.e. on } \Omega\right\} \\
& \leq \sup _{u}\left\{\langle u, \mu\rangle \mid u \in L_{\infty}^{m n}(\Omega), u(x)^{\top} v \leq r(x, v) \forall v \in E^{m n}, \text { a.e. on } \Omega\right\} \\
& \leq \sup _{u}\left\{\langle u, \mu\rangle\left|u \in L_{\infty}^{m n}(\Omega), u(x)^{\top} v \leq \gamma_{2}\right| v \mid \forall v \in E^{m n}, \text { a.e. on } \Omega\right\}
\end{aligned}
$$

and this means $\gamma_{1}\|\mu\| \leq\|\mu\|^{*} \leq \gamma_{2}\|\mu\|$ thus equivalence of both norms.

Now, let $\mathfrak{S}_{0}=\left\{\sigma \in W_{\infty}^{1, n}(\Omega) \mid \sigma(\hat{x})=0\right\}$ and $U$ be a subspace of $L_{\infty}^{m n}(\Omega)$, charactérized by

$$
U:=\left\{u \in L_{\infty}^{m n}(\Omega) \mid u=\nabla \sigma, \sigma \in \mathfrak{S}_{0}\right\}
$$

In virtue of Sóbolev's embedding theorems [3: p. 60], the mapping $f: U \rightarrow \mathbb{R}$ is a linear continuous functional $\mu_{0}$ on $U$, if we define $f(\nabla \sigma):=K_{D}(\sigma)$ for all $\sigma \in \mathfrak{S}_{0}$. Namely, there is a constant $M>0$ such that for every $\sigma$ of this type

$$
\|\sigma\|_{C^{n}(\bar{\Omega})} \leq M \operatorname{ess} \sup _{\Omega}|\nabla \sigma|
$$

holds and therefore

$$
|f(\nabla \sigma)|=\left|K_{D}(\sigma)\right| \leq M \int_{\Omega} d|\alpha|\|\nabla \sigma\|_{L_{\infty}^{m n}(\Omega)}
$$

The linearity of $f$ is obvious. Together with the boundedness (14) of $f$ it follows that $f$ is a linear continuous functional $\mu_{0}$ on $U$. By the Hahn-Banach extension theorem [1: p. 109] we can extend $\mu_{0}$ as a continuous linear functional on the whole space $L_{\infty}^{m n}(\Omega)$ with the same norm. That means, for each $u \in U$ there is uniquely a $\sigma \in \mathfrak{S}_{0}$ such that $u=\nabla \sigma$,

$$
f(u)=K_{D}(\sigma)=\left\langle\nabla \sigma, \mu_{0}\right\rangle
$$

and, with (12),

$$
\left\|\mu_{0}\right\|^{*}=\sup \left\{\left\langle\nabla \sigma, \mu_{0}\right\rangle \mid \sigma \in \mathfrak{S}\right\}=\sup _{\mathfrak{S}} K_{D}=K_{D}\left(S_{0}\right)
$$

hold.

After the extension of $\mu_{0}$ on the totality of $L_{\infty}^{m n}(\Omega)$, it holds again, according to (12),

$$
\left\|\mu_{0}\right\|^{*}=\sup _{u}\left\{\left\langle u, \mu_{0}\right\rangle \mid u \in L_{\infty}^{m n}(\Omega), u(x) \in \mathfrak{F}(x) \text { a.e. on } \Omega\right\}
$$

and since (6), (10) and (16)

$$
\left\|\mu_{0}\right\|^{*}=K\left(\mu_{0}\right)=K_{D}\left(S_{0}\right)
$$

From (15) $\mu_{0} \in Y$ follows such that (8) and (17) lead to the optimality of $\mu_{0}$ with respect to problem (1). So we can summarize:

Theorem 2. The transportation flow problem (1) has an optimal solution $\mu_{0}$. 


\section{Conclusions and generalizations}

The existence of optimal solutions $S_{0}$ of the deposit problem (7) and $\mu_{0}$ of the transportation flow problem (1) has in connection with (8) and (17) the following consequence.

Theorem 3. Between the dual problems (1) and (7) there exists strong duality in the sense that $\min _{Y} K=\max K_{D}$.

From this theorem we obtain under consideration of $(3),(12),(15)$ and (17)

$$
K_{D}\left(S_{0}\right)=\int_{\Omega} S_{0}(x)^{\top} d \alpha(x)=K\left(\mu_{0}\right)=\left\langle\nabla S_{0}, \mu_{0}\right\rangle \geq\left\langle u, \mu_{0}\right\rangle
$$

for all $u \in L_{\infty}^{m n}(\Omega), u(x) \in \mathfrak{F}(x)$ a.c. This leads to the following conclusion.

Theorem 4. An element $S_{0} \in \mathfrak{S}$ is an optimal solution of the deposit problem (7) if and only if there is a vectorial set function $\mu_{0} \in L_{\infty}^{m n}(\Omega)^{*}$ which satisfies the continuity equation

$$
\left\langle\nabla \sigma, \mu_{0}\right\rangle=\int_{\Omega} \sigma(x)^{\top} d \alpha(x) \quad \forall \sigma \in W_{\infty}^{1, n}(\Omega)
$$

and the maximum condition

$$
\left\langle\nabla S_{0}, \mu_{0}\right\rangle \geq\left\langle u, \mu_{0}\right\rangle \quad \forall u \in L_{\infty}^{m n}(\Omega), u(x) \in \mathfrak{F}(x) \text { a.e. on } \Omega \text {. }
$$

Remark. Theorems 3 and 4 coincide essentially with Theorems 4 and 3 from [2]. However, unfortunately the proof of Theorem 3 in that paper was not correct because of a mistake in identifying weak *-compactness and sequentially weak*-compactness by the application of Alaoglu's theorem. Finally, we mention that all results proved here hold also for the case in which $W_{\infty}^{1, n}(\Omega)$ in $(2)$ and $(9)$ is replaced by $W_{\infty}^{1, n}(\Omega)$. Then we can omit even assumption (4).

\section{References}

[1] Kantorowitsch, L. W. and A. P. Akilow: Funktionalanalysis in normierten Räumen. Berlin: Akademie-Verlag 1954.

[2] Klötzler, R.: Optimal transportation flows. Z. Anal. Anw. 14 (1995), $391-401$.

[3] Sobolew, S. L.: Einige Anwendungen der Funktionalanalysis auf Gleichungen der mathematischen Physik. Berlin: A kademie-Verlag 1964. 